\title{
MY-AKKHARA: \\ A Romanization-based Burmese (Myanmar) Input Method
}

\author{
Chenchen Ding, Masao Utiyama, and Eiichiro Sumita \\ Advanced Translation Technology Laboratory, \\ Advanced Speech Translation Research and Development Promotion Center, \\ National Institute of Information and Communications Technology \\ 3-5 Hikaridai, Seika-cho, Soraku-gun, Kyoto, 619-0289, Japan \\ \{chenchen.ding, mutiyama, eiichiro.sumita\}@nict.go.jp
}

\begin{abstract}
MY-AKKHARA is a method used to input Burmese texts encoded in the Unicode standard, based on commonly accepted Latin transcription. By using this method, arbitrary Burmese strings can be accurately inputted with 26 lowercase Latin letters. Meanwhile, the 26 uppercase Latin letters are designed as shortcuts of lowercase letter sequences. The frequency of Burmese characters is considered in MY-AKKHARA to realize an efficient keystroke distribution on a QWERTY keyboard. Given that the Unicode standard has not been extensively used in digitization of Burmese, we hope that MY-AKKHARA can contribute to the widespread use of Unicode in Myanmar and can provide a platform for smart input methods for Burmese in the future. An implementation of MY-AKKHARA running in Windows is released at http: //www2.nict.go.jp/astrec-att/ member/ding/my-akkhara.html
\end{abstract}

\section{Introduction}

Burmese (Myanmar) script is an abugida system, wherein basic characters can be modified using diacritics at all directions or can be combined vertically, rather than a simple left-to-right horizontal writing (Ding et al., 2016). Details of the Burmese language can be referred to in Okell and Allott (2001), Okell (2010a,b), and Okano (2007).

Although its use is encouraged in the government and universities, the use of Unicode for Burmese script ${ }^{1}$ is not currently widespread. Traditional shape-based typefaces such as Zawgyi ${ }^{2}$ are preferred for daily use. The issue can be regarded as path dependence due to traditional typewriters, wherein the input is exactly based

\footnotetext{
${ }^{1}$ https://www. unicode.org/charts/PDF/ $\mathrm{U} 1000 \cdot \mathrm{pdf}$

${ }^{2}$ https://code.google.com/archive/p/ zawgyi/downloads
}

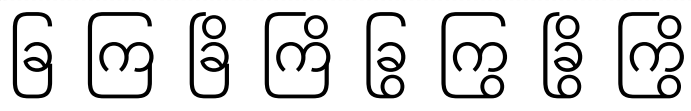

8 shape variants of Zawgyi for Unicode $103 c \subseteq$

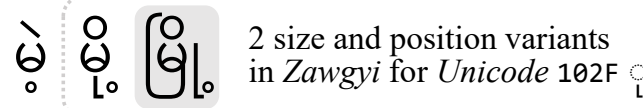

3 position variants in Zawgyi for Unicode 1037

Figure 1: Shape, size, and position variants in Zawgyi for identical Unicode characters. The characters may affect each other: for those with gray background, the shape of $103 \mathrm{C}$ is determined by the inside combination, size and position of $102 \mathrm{~F}$ by $103 \mathrm{C}$, and the position of 1037 by $102 \mathrm{~F}$.

on character shape, rather than the phonetic values of characters (Fig. 1). Zawgyi separately encodes all possible variants of characters and diacritics, and allows users to select correct variants manually. Hence, a redundant character set becomes incompatible with the Unicode standard, and extra effort is required for users to utilize typeface in detail. To provide a better interface and promote Unicode for Burmese digitization, we design a Burmese input method referred to as MY-AKKHARA by Romanization based on the Unicode standard. MY-AKKHARA is generally based on the mnemonics used in Unicode and the Myanmar Language Committee Transcription System (Department of the Myanmar Language Commission, 2014). The efficiency of the key distribution on the QWERTY keyboard layout is also considered in the design of MY-AKKHARA.

The implementation of MY-AKKHARA running in Windows has been released. In this paper, we first review the default layouts of Burmese provided in Windows (Win) and Macintosh (Mac) and subsequently provide detailed descriptions of MY-AKKHARA. In addition, the keystroke distribution of different methods is compared. 


\section{Win and Mac Burmese Keyboards}

The keyboard layouts used to input Burmese in Unicode have been provided in Win and Mac operating systems. Figure 2 illustrates the default layouts of the Burmese Unicode keyboard in these two mainstream operating systems. Both of the layouts are a simple mapping from characters to keys. Excluding special punctuation marks and native number digits, 63 Unicode characters are required to represent modern standard Burmese textual data, from Unicode 1000 to $104 \mathrm{~F} .{ }^{3}$ Therefore, 26 keys with shift are not sufficient to cover the character set. In both of the layouts, extra punctuation (or digit), or alternative keys are necessary in typing.

The Win layout is adjusted from the traditional layout of a typewriter, by removing redundant character varieties and re-arranging the characters inputted using the Shift-key. Considering that this layout has a large portion of the traditional one but with a certain difference, many Win users are not interested in switching to this layout. Hence non-Unicode fonts are still inputted using the traditional keyboard in practice. Moreover, the Mac layout is completely redesigned based on Romanization manner, wherein the Burmese characters are arranged on the basis of their pronunciations as represented by the letters on a QWERTY keyboard. However, the design is inflexible, without considering the practical use of Burmese characters. Thus, the positioning of fingers when typing is tricky. The comparison of the keystroke distribution will be presented in Section 4 .

\section{MY-AKKHARA: Proposed Input Method}

The proposed MY-AKKHARA is inspired by the Mac layout and deemed highly natural and efficient. Rather than a simple mapping between the Unicode characters and keys, we also facilitate character alternation processing by using the inputted Latin letters. Specifically, double keystrokes of e, f, h, i, j, r, u, v, w, and y, and the $h$ - and g-keys at the middle of a QWERTY keyboard are used to alternate characters. This design naturally integrates the Romanization into the character alternation processing. The q-key is reserved to disambiguate in obscure cases through which the input method can precisely input any

\footnotetext{
${ }^{3}$ Within this range, from 1040 to $104 \mathrm{~B}$ are Burmese digits and punctuation marks; 1022, 1028, 1033, 1034, and 1035 are not used for standard Burmese.
}

strings with the Unicode Burmese characters. ${ }^{4}$ Lowercase $\mathrm{a}, \mathrm{o}, \mathrm{x}$, and 26 uppercase Latin letters are assigned as optional shortcuts. Figure 3 shows an example on the technique of inputting a Burmese string with rare and stacked characters using the proposed method.

The instruction of the proposed input method can be printed by users on an A4 paper (Fig. 4). The proposed method can be formulated primarily through a finite-state automaton (Hopcroft et al., 2013), receiving strings comprising 23 lowercase Latin letters (excluding $a, 0$, and $\mathrm{x}$ ) and transiting among different states that represent Burmese characters. The Appendix provides the description of the automaton.

The shortcuts can be grouped in the following four categories:

- three lowercase letters for common combinations: $a=q e v q, o=q i u q$, and $x=q n g f q$;

- uppercase letters to save double keystrokes: $E=q e e, \quad F=q f f, \quad H=q h h, \quad I=q i i, \quad J=q j j$, $R=q r r, U=q u u, V=q v v, W=q W W$, and $Y=q Y Y$;

- uppercase letters to save $\mathrm{h} / \mathrm{g}: \mathrm{B}=\mathrm{qbh}, \mathrm{C}=\mathrm{qch}$, $\mathrm{D}=\mathrm{qdh}, \mathrm{G}=\mathrm{qgh}, \mathrm{K}=\mathrm{qkh}, \mathrm{L}=\mathrm{ql} \mathrm{g}, \mathrm{M}=\mathrm{qmg}$, $\mathrm{P}=q \mathrm{ph}, \mathrm{Q}=\mathrm{qg}, \mathrm{T}=\mathrm{qt} h$, and $\mathrm{Z}=\mathrm{qzh}$; and

- uppercase letters for other cases: $A=q e g g$, $\mathrm{N}=\mathrm{qny}, \mathrm{O}=\mathrm{qs} \mathrm{r}, \mathrm{S}=$ quug, and $\mathrm{X}=\mathrm{qng}$.

Lowercase letters $a, 0$, and $x$ can considerably save keystrokes. Note that the shortcuts have a preceding $q$ in the implementation through which disambiguation can be realized. The recommended uppercase letters are $\mathrm{Y}, \mathrm{H}$, and $\mathrm{Q}$, which can resolve almost all ambiguous cases when typing orthographically correct Burmese texts.

Two issues related to normalizing the encoding of the Burmese script in Unicode are addressed:

- $102 \mathrm{~B}$ is a variant of $102 \mathrm{C}$, exclusively used for narrow characters of $1001,1002,1004$, 1012,1015 , and 101D. This alternation is executed automatically when typing $\mathrm{v}$ or a (i.e., shortcut for ev). However, qv and qvg can exactly input $102 \mathrm{C}$ and $102 \mathrm{~B}$, respectively.

- 1037 and 103A can appear successively; however, their order is not precisely identified. 103A 1037 will always be normalized in Unicode into the recommended order 1037 103A.

\footnotetext{
${ }^{4}$ It is possible to intentionally input orthographically incorrect Burmese strings; however, orthographically correct strings can be inputted more naturally than incorrect ones.
} 

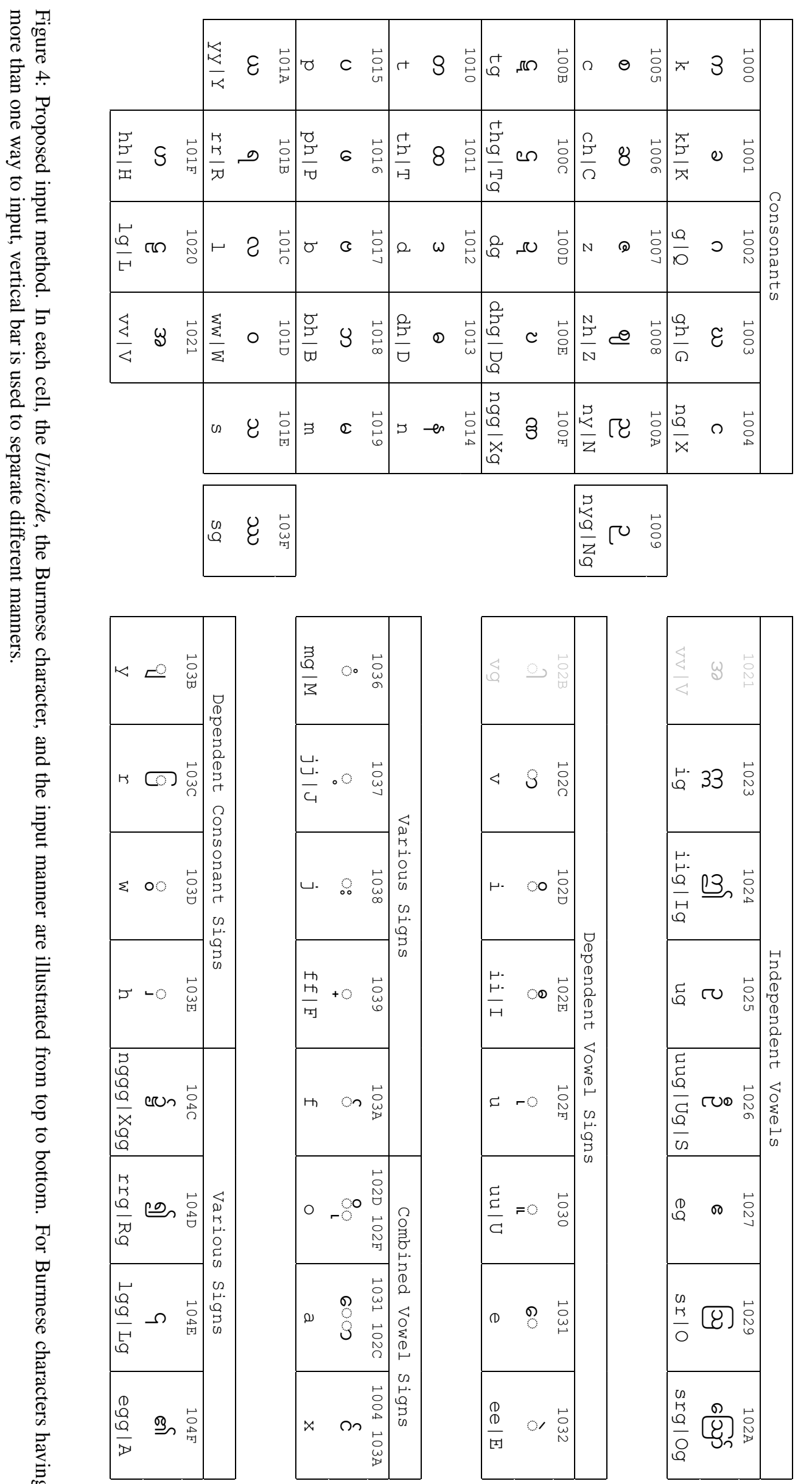

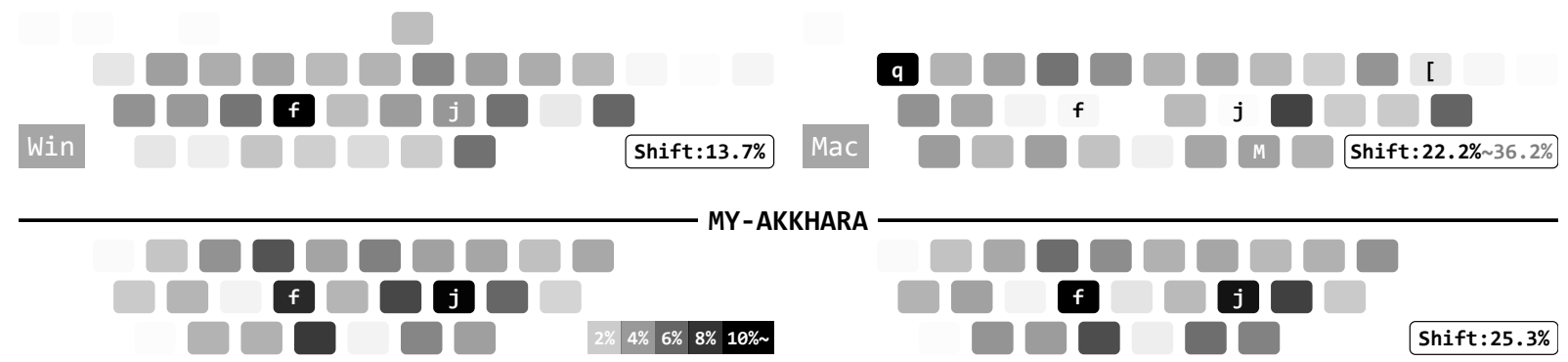

Figure 5: Keystroke distribution on the ALT literary data. The upper-left and upper-right diagrams are Win and Mac keyboards, respectively. The lower images are MY-AKKHARA, with Shift not used (left) and Shift completely used (right) manners, respectively. The usage frequency of the Shift-key is also presented. Note that $103 \mathrm{~A}$ and 1036 appear twice on the Mac keyboard. The two characters are counted by using $q$ and [ to input in the diagram, where the frequency of Shift-key is $22.2 \%$. The two character can be also inputted by uppercase $\mathrm{F}$ and M. If they are always inputted using the Shift-key, then the frequency of Shift-key increases to $36.2 \%$.

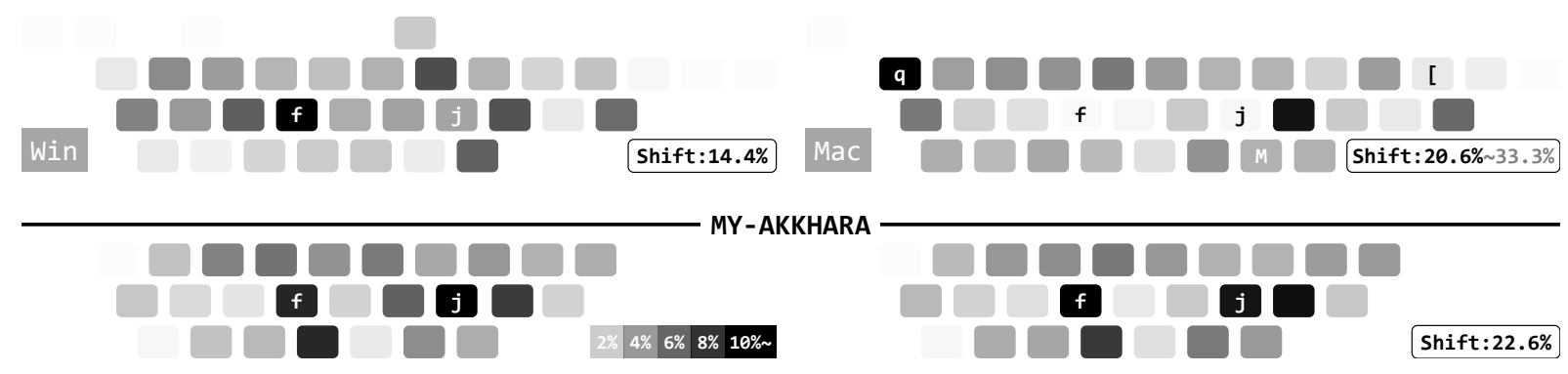

Figure 6: Keystroke distribution on the BTEC colloquial data. The configuration is the same as that of Fig. 5.

Chenchen Ding, Ye Kyaw Thu, Masao Utiyama, and Eiichiro Sumita. 2016. Word segmentation for Burmese (Myanmar). ACM TALLIP, 15(4):22.

John E. Hopcroft, Rajeev Motwani, and Jeffrey D. Ullman. 2013. Introduction to Automata Theory, Languages, and Computation, 3 edition. Pearson.

Genichiro Kikui, Eiichiro Sumita, Toshiyuki Takezawa, and Seiichi Yamamoto. 2003. Creating corpora for speech-to-speech translation. In Proc. of EUROSPEECH, pages 381-384.

Kenji Okano. 2007. Colloquial Burmese (Myanmar) Grammar. Kokusai Gogakusha. (in Japanese).

John Okell. 2010a. Burmese - An introduction to the Spoken Language, Book 1. Northern Illinois University Press.

John Okell. 2010b. Burmese - An introduction to the Spoken Language, Book 2. Northern Illinois University Press.

John Okell and Anna Allott. 2001. Burmese / Myanmar Dictionary of Grammatical Forms. Routledge.

Hammam Riza, Michael Purwoadi, Teduh Uliniansyah, Aw Ai Ti, Sharifah Mahani Aljunied, Luong Chi Mai, Vu Tat Thang, Nguyen Phuong Thai, Vichet Chea, Rapid Sun, Sethserey Sam, Sopheap Seng, Khin Mar Soe, Khin Thandar Nwet, Masao Utiyama, and Chenchen Ding. 2016. Introduction of the Asian language treebank. In Proc. of $\mathrm{O}$ COCOSDA, pages 1-6.

\section{Appendix}

Figure 7 shows the overall configuration. Routes connecting the the initial $\left(q_{s}\right)$ and final $\left(q_{s}\right)$ states are listed in Figs. $8-16$, where $q_{n},(n \in \mathbf{N})$ are Burmese characters. ${ }^{6}$ Although all $q_{n}$ can be the final states, a separate $q_{e}$ is used for clarity, and a $q$ is marked explicitly on all the arcs to $q_{e}$.

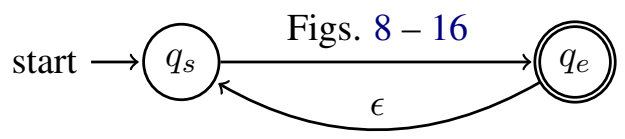

Figure 7: Overall configuration of the automaton.

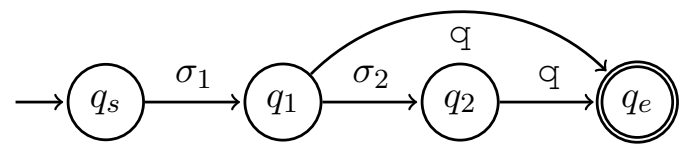

Figure 8: Simplest case. When $\sigma_{2}$ is $\mathrm{h},\left(\sigma_{1}, q_{1}, q_{2}\right)$ can be $(\mathrm{k}, 00,01),(\mathrm{g}, 02,03),(\mathrm{c}, 05,06)$, $(\mathrm{z}, 07,08),(\mathrm{p}, 15,16)$, and $(\mathrm{b}, 17,18)$. When $\sigma_{2}$ is $\mathrm{g},\left(\sigma_{1}, q_{1}, q_{2}\right)$ is (m, 19, 36). When $\sigma_{2}=\sigma_{1}$, $\left(\sigma_{1}, q_{1}, q_{2}\right)$ can be $(\mathrm{y}, 3 \mathrm{~B}, 1 \mathrm{~A}),(\mathrm{w}, 3 \mathrm{D}, 1 \mathrm{C})$, and (h, 3E, 1D). All $\sigma_{1}$ are natural Romanization. When $\sigma_{2}$ is $h$, it is also a part of the Romanization.

\footnotetext{
${ }^{6}$ Unicode is referred to by the final two digits for brevity.
} 


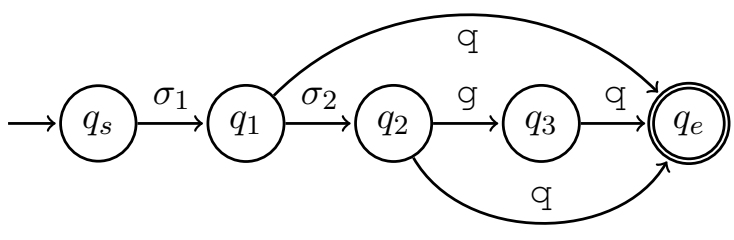

Figure 9: Two-step alternation. When $\left(\sigma_{1}, \sigma_{2}\right)$ is $(1, g),\left(q_{1}, q_{2}, q_{3}\right)$ is $(1 \mathrm{C}, 20,4 \mathrm{E})$. Here, 1 is a natural Romanization for $101 \mathrm{C}$ and 1020 , whereas $104 \mathrm{E}$ is a special abbreviated mark with 1 as onset. When $\left(\sigma_{1}, \sigma_{2}\right)$ is $(r, r),\left(q_{1}, q_{2}, q_{3}\right)$ is (3C, $\left.1 \mathrm{~B}, 4 \mathrm{D}\right)$, respectively. Here, $r$ is a natural Romanization for $103 \mathrm{C}$ and $101 \mathrm{~B}$, whereas $104 \mathrm{D}$ is a special abbreviated mark with $r$ as onset.

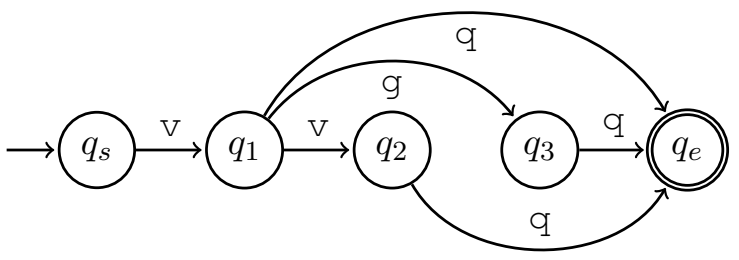

Figure 10: Alternation variant of Fig. 9. $\left(q_{1}, q_{2}, q_{3}\right)$ is $(2 \mathrm{C}, 21,2 \mathrm{~B})$. Considering that $102 \mathrm{C}$ and 1021 are frequently used, the convenient $\mathrm{v}$-key is assigned instead the natural Romanization by $a$.

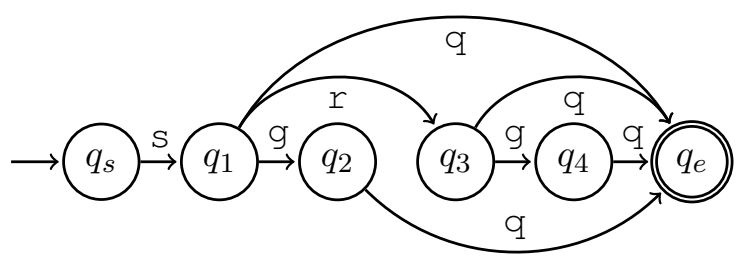

Figure 11: Alternation in Fig. 8 with an extra branch. $\left(q_{1}, q_{2}, q_{3}, q_{4}\right)$ is (1E, 3F, 29, 2A). Here, $\mathrm{s}$ is a natural Romanization for $101 \mathrm{E}$, whereas $103 \mathrm{~F}$, 1029 , and 102A are extremely obscure.

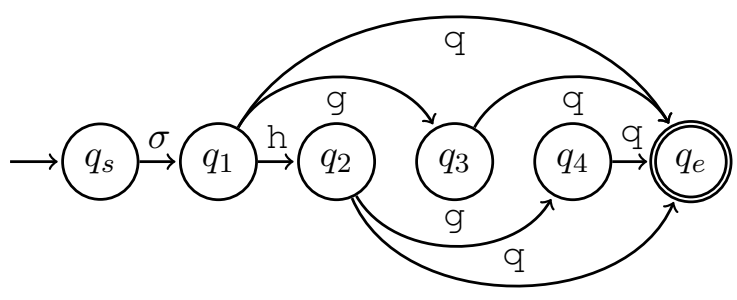

Figure 12: Alternation by $\mathrm{h}$ and g. $\left(\sigma, q_{1}, q_{2}, q_{3}, q_{4}\right)$ can be (t, 10, 11, OB, OC), and (d, 12, 13, OD, OE). Both $t$ and $d$ are the natural Romanization, and $h$ is also a part of the Romanization.

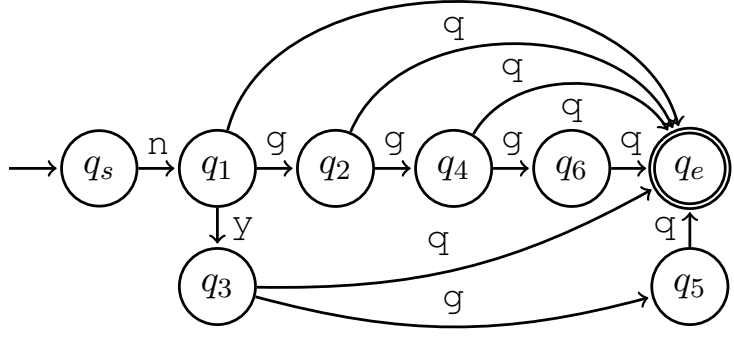

Figure 13: Most complex alternation.

$\left(q_{1}, q_{2}, q_{3}, q_{4}, q_{5}, q_{6}\right)$ is $(14,04,0 \mathrm{~A}, 0 \mathrm{~F}, 09,4 \mathrm{C})$.

Here, n, ng and ny are the natural Romanization for 1014,1004 , and 100A, respectively. Other alternated characters are rare.

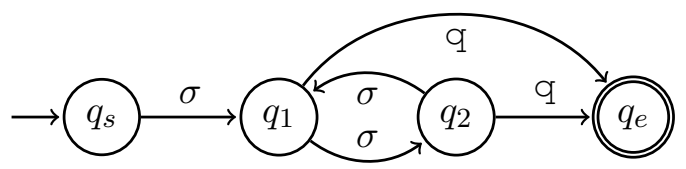

Figure 14: Doubled and looped alternation. $\left(\sigma, q_{1}, q_{2}\right)$ can be (j, 38, 37), and (f, 3A, 39). Here, 1038 and $103 \mathrm{~A}$ are remarkably frequent marks; hence convenient $j$ - and $f$-keys are assigned, respectively.

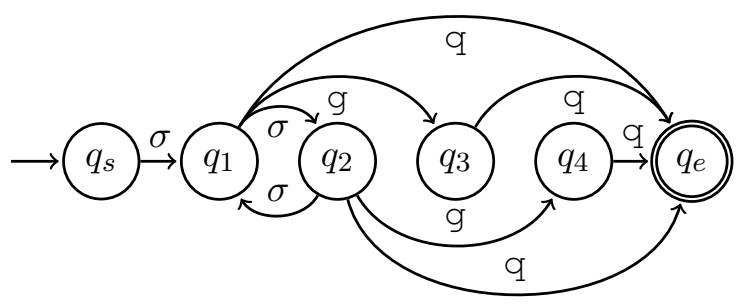

Figure 15: Combination of Figs. 12 and 14. $\left(\sigma, q_{1}, q_{2}, q_{3}, q_{4}\right)$ can be (i, 2D, 2E, 23, 24), and ( $u, 2 F, 30,25,26)$. Here, $i$ and $u$ are the natural Romanization for the corresponding characters.

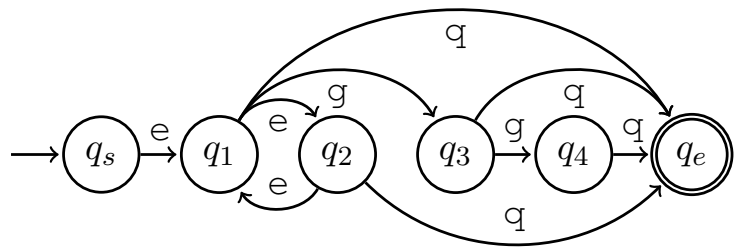

Figure 16: Alternation in Fig. 14 with an extra branch. $\left(q_{1}, q_{2}, q_{3}, q_{4}\right)$ is $(31,32,27,4 \mathrm{~F})$. Here, e is a natural Romanization for 1031, 1032 and 1027, whereas $104 \mathrm{~F}$ is an abbreviated mark derived from 1027 . 